\title{
Existence of positive solutions of the Cauchy problem for a second-order differential equation
}

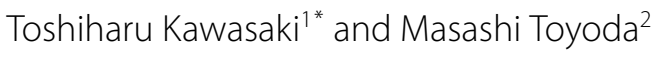

\section{"Correspondence:}

toshiharu.kawasaki@nifty.ne.jp

${ }^{1}$ College of Engineering, Nihon University, Fukushima, 963-8642, Japan

Full list of author information is available at the end of the article

\section{Abstract}

In this paper we consider the equation $u^{\prime \prime}(t)=f\left(t, u(t), u^{\prime}(t)\right)$ and prove the unique solvability of the Cauchy problem $u(0)=0, u^{\prime}(0)=\lambda$ with $\lambda>0$.

\section{Introduction}

In [1], Knežević-Miljanović considered the Cauchy problem

$$
\left\{\begin{array}{l}
u^{\prime \prime}(t)=P(t) t^{a} u(t)^{\sigma}, \quad t \in(0,1] \\
u(0)=0, \quad u^{\prime}(0)=\lambda
\end{array}\right.
$$

where $P$ is continuous, $a, \sigma, \lambda \in \mathbb{R}$ with $\sigma<0$ and $\lambda>0$, and $\int_{0}^{1}|P(t)| t^{a+\sigma} d t<\infty$. Moreover, in [2], Kawasaki and Toyoda considered the Cauchy problem

$$
\left\{\begin{array}{l}
u^{\prime \prime}(t)=f(t, u(t)), \quad \text { for almost every } t \in[0,1], \\
u(0)=0, \quad u^{\prime}(0)=\lambda,
\end{array}\right.
$$

where $f$ is a mapping from $[0,1] \times(0, \infty)$ into $\mathbb{R}$ and $\lambda \in \mathbb{R}$ with $\lambda>0$. They proved the unique solvability of Cauchy problem (2) using the Banach fixed point theorem. The theorem in [2] is as follows.

Theorem Suppose that a mapping f from $[0,1] \times[0, \infty)$ into $\mathbb{R}$ satisfies the following.

(a) The mapping $t \longmapsto f(t, u)$ is measurable for any $u \in(0, \infty)$, and the mapping $u \longmapsto f(t, u)$ is continuous for almost every $t \in[0,1]$.

(b) $\left|f\left(t, u_{1}\right)\right| \geq\left|f\left(t, u_{2}\right)\right|$ for almost every $t \in[0,1]$ and for any $u_{1}, u_{2} \in[0, \infty)$ with $u_{1} \leq u_{2}$.

(c) There exists $\alpha \in \mathbb{R}$ with $0<\alpha<\lambda$ such that

$$
\int_{0}^{1}|f(t, \alpha t)| d t<\infty
$$

(d) There exists $\beta \in \mathbb{R}$ with $\beta>0$ such that

$$
\left|\frac{\partial f}{\partial u}(t, u)\right| \leq \frac{\beta|f(t, u)|}{u}
$$

for almost every $t \in[0,1]$ and for any $u \in(0, \infty)$. 
Then there exists $h \in \mathbb{R}$ with $0<h \leq 1$ such that Cauchy problem (2) has a unique solution in $X$, where $X$ is a subset

$$
X=\left\{\begin{array}{l|l}
u & \begin{array}{l}
u \in C[0, h], u(0)=0, u^{\prime}(0)=\lambda \\
\text { and } \alpha t \leq u(t) \text { for any } t \in[0, h]
\end{array}
\end{array}\right\}
$$

of $C[0, h]$, which is the class of continuous mappings from $[0, h]$ into $\mathbb{R}$.

The case that $f(t, u(t))=P(t) t^{a} u(t)^{\sigma}$ in the above theorem is the theorem of KneževićMiljanović [1].

In this paper, we consider the Cauchy problem

$$
\left\{\begin{array}{l}
u^{\prime \prime}(t)=f\left(t, u(t), u^{\prime}(t)\right), \quad \text { for almost every } t \in[0,1] \\
u(0)=0, \quad u^{\prime}(0)=\lambda,
\end{array}\right.
$$

where $f$ is a mapping from $[0,1] \times(0, \infty) \times \mathbb{R}$ into $\mathbb{R}$ and $\lambda \in \mathbb{R}$ with $\lambda>0$. We prove the unique solvability of this Cauchy problem using the Banach fixed point theorem.

In Section 2, we consider the following four cases for $u$ and $v$.

(I) Decreasing for $u$ in $f(t, u, v)$ (b1) and decreasing for $v$ in $f(t, u, v)$ (b3).

(II) Decreasing for $u$ in $f(t, u, v)$ (b1) and increasing for $v$ in $f(t, u, v)$ (b4).

(III) Increasing for $u$ in $f(t, u, v)$ (b2) and decreasing for $v$ in $f(t, u, v)$ (b3).

(IV) Increasing for $u$ in $f(t, u, v)$ (b2) and increasing for $v$ in $f(t, u, v)$ (b4).

Theorems 2.1, 2.2, 2.3 and 2.4 are the cases of (I), (II), (III) and (IV), respectively.

\section{Main results}

In this section, we consider the Cauchy problem

$$
\left\{\begin{array}{l}
u^{\prime \prime}(t)=f\left(t, u(t), u^{\prime}(t)\right), \quad \text { for almost every } t \in[0,1] \\
u(0)=0, \quad u^{\prime}(0)=\lambda,
\end{array}\right.
$$

where $f$ is a mapping from $[0,1] \times(0, \infty) \times \mathbb{R}$ into $\mathbb{R}$ and $\lambda \in \mathbb{R}$ with $\lambda>0$.

First, we consider the case of (I).

Theorem 2.1 Let $\lambda$ be a real number with $\lambda>0$. Suppose that a mapping $f$ from $[0,1] \times$ $(0, \infty) \times \mathbb{R}$ into $\mathbb{R}$ satisfies the following:

(a) The mapping $t \longmapsto f(t, u, v)$ is measurable for any $(u, v) \in(0, \infty) \times \mathbb{R}$, and the mapping $(u, v) \longmapsto f(t, u, v)$ is continuous for almost every $t \in[0,1]$;

(b1) $\left|f\left(t, u_{1}, v\right)\right| \geq\left|f\left(t, u_{2}, v\right)\right|$ for almost every $t \in[0,1]$, for any $u_{1}, u_{2} \in(0, \infty)$ with $u_{1} \leq u_{2}$ and for any $v \in \mathbb{R}$

(b3) $\left|f\left(t, u, v_{1}\right)\right| \geq\left|f\left(t, u, v_{2}\right)\right|$ for almost every $t \in[0,1]$, for any $u \in(0, \infty)$ and for any $v_{1}, v_{2} \in \mathbb{R}$ with $v_{1} \leq v_{2}$

(c1) There exist $\alpha_{1} \in \mathbb{R}$ with $0<\alpha_{1}<\lambda$ and $\alpha_{2} \in \mathbb{R}$ with $\alpha_{2}<\lambda$ such that

$$
\int_{0}^{1}\left|f\left(t, \alpha_{1} t, \alpha_{2}\right)\right| d t<\infty
$$


(d1) There exists $\beta_{1} \in \mathbb{R}$ with $\beta_{1}>0$ such that

$$
\left|\frac{\partial f}{\partial u}(t, u, v)\right| \leq \frac{\beta_{1}|f(t, u, v)|}{u}
$$

for almost every $t \in[0,1]$, for any $u \in(0, \infty)$ and for any $v \in \mathbb{R}$;

(d2) There exists $\beta_{2} \in \mathbb{R}$ with $\beta_{2}>0$ such that

$$
\left|\frac{\partial f}{\partial v}(t, u, v)\right| \leq \beta_{2}|f(t, u, v)|
$$

for almost every $t \in[0,1]$, for any $u \in(0, \infty)$ and for any $v \in \mathbb{R}$;

(e) There exists the limit

$$
\lim _{t \rightarrow 0+} \frac{1}{t^{2}} \int_{0}^{t} s f\left(s, u(s), u^{\prime}(s)\right) d s
$$

for any continuously differentiable mapping $u$ from $[0,1]$ into $[0, \infty)$;

(f1) For $\alpha_{1}$ and $\alpha_{2}$,

$$
\lim _{t \rightarrow 0+} \frac{1}{t^{2}} \int_{0}^{t} s\left|f\left(s, \alpha_{1} s, \alpha_{2}\right)\right| d s=0
$$

Then there exists $h \in \mathbb{R}$ with $0<h \leq 1$ such that Cauchy problem (3) has a unique solution in $X$, where $X$ is a subset

$$
X=\left\{\begin{array}{l|l}
u & \begin{array}{l}
u \in C^{1}[0, h], u(0)=0, u^{\prime}(0)=\lambda, \\
\alpha_{1} t \leq u(t) \text { and } \alpha_{2} \leq u^{\prime}(t) \text { for any } t \in[0, h] \\
\text { and there exists the limit } \lim _{t \rightarrow 0+} \frac{t u^{\prime}(t)-u(t)}{t^{2}}
\end{array}
\end{array}\right\}
$$

of $C^{1}[0, h]$, which is the class of continuously differentiable mappings from $[0, h]$ into $\mathbb{R}$.

Proof It is noted that $C^{1}[0, h]$ is a Banach space by the maximum norm

$$
\|u\|=\max \left\{\max \{|u(t)| \mid t \in[0, h]\}, \max \left\{\left|u^{\prime}(t)\right| \mid t \in[0, h]\right\}\right\} .
$$

Instead of Cauchy problem (3), we consider the integral equation

$$
u(t)=\lambda t+\int_{0}^{t}(t-s) f\left(s, u(s), u^{\prime}(s)\right) d s
$$

By condition (c1), there exists $h_{1} \in \mathbb{R}$ with $0<h_{1} \leq 1$ such that

$$
\int_{0}^{h_{1}}\left|f\left(t, \alpha_{1} t, \alpha_{2}\right)\right| d t<\min \left\{\lambda-\alpha_{1}, \lambda-\alpha_{2},\left(\frac{\beta_{1}}{\alpha_{1}}+2 \beta_{2}\right)^{-1}\right\} .
$$

By condition (f1), there exists $h \in \mathbb{R}$ with $0<h \leq h_{1}$ such that

$$
\sup _{t \in(0, h]} \frac{1}{t^{2}} \int_{0}^{t} s\left|f\left(s, \alpha_{1} s, \alpha_{2}\right)\right| d s \leq \int_{0}^{h_{1}}\left|f\left(t, \alpha_{1} t, \alpha_{2}\right)\right| d t .
$$


Let $A$ be an operator from $X$ into $C^{1}[0, h]$ defined by

$$
A u(t)=\lambda t+\int_{0}^{t}(t-s) f\left(s, u(s), u^{\prime}(s)\right) d s .
$$

Since a mapping $t \longmapsto \lambda t$ belongs to $X, X \neq \emptyset$. Moreover, we have $A(X) \subset X$. Indeed, by condition (a), $A u \in C^{1}[0, h], A u(0)=0$ and

$$
(A u)^{\prime}(0)=\left[\lambda+\int_{0}^{t} f\left(s, u(s), u^{\prime}(s)\right) d s\right]_{t=0}=\lambda .
$$

By conditions (b1) and (b3), we obtain that

$$
\begin{aligned}
A u(t) & =\lambda t+\int_{0}^{t}(t-s) f\left(s, u(s), u^{\prime}(s)\right) d s \\
& \geq \lambda t-t \int_{0}^{h}\left|f\left(s, u(s), u^{\prime}(s)\right)\right| d s \\
& \geq \lambda t-t \int_{0}^{h}\left|f\left(s, \alpha_{1} s, \alpha_{2}\right)\right| d s \\
& \geq \alpha_{1} t
\end{aligned}
$$

and

$$
\begin{aligned}
(A u)^{\prime}(t) & =\lambda+\int_{0}^{t} f\left(s, u(s), u^{\prime}(s)\right) d s \\
& \geq \lambda-\int_{0}^{h}\left|f\left(s, u(s), u^{\prime}(s)\right)\right| d s \\
& \geq \lambda-\int_{0}^{h}\left|f\left(s, \alpha_{1} s, \alpha_{2}\right)\right| d s \\
& \geq \alpha_{2}
\end{aligned}
$$

for any $t \in[0, h]$. Moreover, by condition (e), there exists the limit

$$
\lim _{t \rightarrow 0+} \frac{t(A u)^{\prime}(t)-A u(t)}{t^{2}}=\lim _{t \rightarrow 0+} \frac{1}{t^{2}} \int_{0}^{t} s f\left(s, u(s), u^{\prime}(s)\right) d s .
$$

We will find a fixed point of $A$. Let $\varphi$ be an operator from $X$ into $C^{1}[0, h]$ defined by

$$
\varphi[u](t)= \begin{cases}\frac{u(t)}{t} & \text { if } t \in(0, h] \\ \lambda & \text { if } t=0\end{cases}
$$

Let $\varphi[X]$ be a subset defined by

$$
\varphi[X]=\{\varphi[u] \mid u \in X\}
$$

Then we have

$$
\varphi[X]=\left\{\begin{array}{l|l}
v & \begin{array}{l}
v \in C^{1}[0, h], v(0)=\lambda, \\
\alpha_{1} \leq v(t) \text { and } \alpha_{2} \leq v(t)+t v^{\prime}(t) \text { for any } t \in[0, h]
\end{array}
\end{array}\right\}
$$


and $\varphi[X]$ is a closed subset of $C^{1}[0, h]$. Hence it is a complete metric space. Let $\Phi$ be an operator from $\varphi[X]$ into $\varphi[X]$ defined by

$$
\Phi \varphi[u]=\varphi[A u] .
$$

By the mean value theorem, for any $u_{1}, u_{2} \in X$, there exist mappings $\xi, \eta$ such that

$$
\begin{aligned}
& f(\left.t, u_{1}(t), u_{1}^{\prime}(t)\right)-f\left(t, u_{2}(t), u_{2}^{\prime}(t)\right) \\
&= \frac{\partial f}{\partial u}\left(t, \xi(t), u_{1}^{\prime}(t)\right)\left(u_{1}(t)-u_{2}(t)\right)+\frac{\partial f}{\partial v}\left(t, u_{2}(t), \eta(t)\right)\left(u_{1}^{\prime}(t)-u_{2}^{\prime}(t)\right) \\
&=\left(t \frac{\partial f}{\partial u}\left(t, \xi(t), u_{1}^{\prime}(t)\right)+\frac{\partial f}{\partial v}\left(t, u_{2}(t), \eta(t)\right)\right)\left(\varphi\left[u_{1}\right](t)-\varphi\left[u_{2}\right](t)\right) \\
&+t \frac{\partial f}{\partial v}\left(t, u_{2}(t), \eta(t)\right)\left(\varphi\left[u_{1}\right]^{\prime}(t)-\varphi\left[u_{2}\right]^{\prime}(t)\right), \\
& \min \left\{u_{1}(t), u_{2}(t)\right\} \leq \xi(t) \leq \max \left\{u_{1}(t), u_{2}(t)\right\}
\end{aligned}
$$

and

$$
\min \left\{u_{1}^{\prime}(t), u_{2}^{\prime}(t)\right\} \leq \eta(t) \leq \max \left\{u_{1}^{\prime}(t), u_{2}^{\prime}(t)\right\}
$$

for almost every $t \in[0, h]$. Therefore, by conditions (b1), (b3), (d1) and (d2), we obtain that

$$
\begin{aligned}
\mid f( & \left.t, u_{1}(t), u_{1}^{\prime}(t)\right)-f\left(t, u_{2}(t), u_{2}^{\prime}(t)\right) \mid \\
= & \mid\left(t \frac{\partial f}{\partial u}\left(t, \xi(t), u_{1}^{\prime}(t)\right)+\frac{\partial f}{\partial v}\left(t, u_{2}(t), \eta(t)\right)\right)\left(\varphi\left[u_{1}\right](t)-\varphi\left[u_{2}\right](t)\right) \\
& +t \frac{\partial f}{\partial v}\left(t, u_{2}(t), \eta(t)\right)\left(\varphi\left[u_{1}\right]^{\prime}(t)-\varphi\left[u_{2}\right]^{\prime}(t)\right) \mid \\
\leq & \left(t\left|\frac{\partial f}{\partial u}\left(t, \xi(t), u_{1}^{\prime}(t)\right)\right|+\left|\frac{\partial f}{\partial v}\left(t, u_{2}(t), \eta(t)\right)\right|\right)\left|\varphi\left[u_{1}\right](t)-\varphi\left[u_{2}\right](t)\right| \\
& +t\left|\frac{\partial f}{\partial v}\left(t, u_{2}(t), \eta(t)\right)\right|\left|\left(\varphi\left[u_{1}\right]^{\prime}(t)-\varphi\left[u_{2}\right]^{\prime}(t)\right)\right| \\
\leq & \left(\frac{\beta_{1}}{\alpha_{1}}+\beta_{2}\right)\left|f\left(t, \alpha_{1} t, \alpha_{2}\right)\right|\left|\varphi\left[u_{1}\right](t)-\varphi\left[u_{2}\right](t)\right| \\
& +\beta_{2} t\left|f\left(t, \alpha_{1} t, \alpha_{2}\right)\right|\left|\left(\varphi\left[u_{1}\right]^{\prime}(t)-\varphi\left[u_{2}\right]^{\prime}(t)\right)\right|
\end{aligned}
$$

for almost every $t \in[0, h]$. Therefore we have

$$
\begin{aligned}
\mid \Phi \varphi & {\left[u_{1}\right](t)-\Phi \varphi\left[u_{2}\right](t) \mid } \\
& =\left|\frac{1}{t} \int_{0}^{t}(t-s)\left(f\left(s, u_{1}(s), u_{1}^{\prime}(s)\right)-f\left(s, u_{2}(s), u_{2}^{\prime}(s)\right)\right) d s\right| \\
& \leq \int_{0}^{t}\left|f\left(s, u_{1}(s), u_{1}^{\prime}(s)\right)-f\left(s, u_{2}(s), u_{2}^{\prime}(s)\right)\right| d s \\
& \leq \int_{0}^{t}\left[\left(\frac{\beta_{1}}{\alpha_{1}}+\beta_{2}\right)\left|f\left(s, \alpha_{1} s, \alpha_{2}\right)\right|\left|\varphi\left[u_{1}\right](s)-\varphi\left[u_{2}\right](s)\right|\right.
\end{aligned}
$$




$$
\begin{aligned}
& \left.+\beta_{2} s\left|f\left(s, \alpha_{1} s, \alpha_{2}\right)\right|\left|\left(\varphi\left[u_{1}\right]^{\prime}(s)-\varphi\left[u_{2}\right]^{\prime}(s)\right)\right|\right] d s \\
\leq & \left(\frac{\beta_{1}}{\alpha_{1}}+2 \beta_{2}\right) \int_{0}^{h}\left|f\left(s, \alpha_{1} s, \alpha_{2}\right)\right| d s\left\|\varphi\left[u_{1}\right]-\varphi\left[u_{2}\right]\right\|
\end{aligned}
$$

for any $t \in[0, h]$. Moreover, we have

$$
\begin{aligned}
\left|\left(\Phi \varphi\left[u_{1}\right]\right)^{\prime}(t)-\left(\Phi \varphi\left[u_{2}\right]\right)^{\prime}(t)\right| \\
=\left|\frac{1}{t^{2}} \int_{0}^{t} s\left(f\left(s, u_{1}(s), u_{1}^{\prime}(s)\right)-f\left(s, u_{2}(s), u_{2}^{\prime}(s)\right)\right) d s\right| \\
\leq \frac{1}{t^{2}} \int_{0}^{t} s\left|f\left(s, u_{1}(s), u_{1}^{\prime}(s)\right)-f\left(s, u_{2}(s), u_{2}^{\prime}(s)\right)\right| d s \\
\leq \frac{1}{t^{2}} \int_{0}^{t} s\left[\left(\frac{\beta_{1}}{\alpha_{1}}+\beta_{2}\right)\left|f\left(s, \alpha_{1} s, \alpha_{2}\right)\right|\left|\varphi\left[u_{1}\right](s)-\varphi\left[u_{2}\right](s)\right|\right. \\
\left.\quad+\beta_{2} s\left|f\left(s, \alpha_{1} s, \alpha_{2}\right)\right|\left|\left(\varphi\left[u_{1}\right]^{\prime}(s)-\varphi\left[u_{2}\right]^{\prime}(s)\right)\right|\right] d s \\
\leq \\
\quad\left[\left(\frac{\beta_{1}}{\alpha_{1}}+\beta_{2}\right) \int_{0}^{h_{1}}\left|f\left(s, \alpha_{1} s, \alpha_{2}\right)\right| d s\right. \\
\left.\quad+\beta_{2} \int_{0}^{h}\left|f\left(s, \alpha_{1} s, \alpha_{2}\right)\right| d s\right]\left\|\varphi\left[u_{1}\right]-\varphi\left[u_{2}\right]\right\|
\end{aligned}
$$

for any $t \in[0, h]$. Hence we obtain that

$$
\begin{aligned}
& \left\|\Phi \varphi\left[u_{1}\right]-\Phi \varphi\left[u_{2}\right]\right\| \\
& \quad \leq\left(\frac{\beta_{1}}{\alpha_{1}}+2 \beta_{2}\right) \int_{0}^{h_{1}}\left|f\left(s, \alpha_{1} s, \alpha_{2}\right)\right| d s\left\|\varphi\left[u_{1}\right]-\varphi\left[u_{2}\right]\right\| .
\end{aligned}
$$

By the Banach fixed point theorem, there exists a unique mapping $\varphi[u] \in \varphi[X]$ such that $\Phi \varphi[u]=\varphi[u]$. Then $A u=u . u$ is a solution of (3).

Next, we consider the case of (II).

Theorem 2.2 Let $\lambda$ be a real number with $\lambda>0$. Suppose that a mapping $f$ from $[0,1] \times$ $(0, \infty) \times \mathbb{R}$ into $\mathbb{R}$ satisfies the following:

(a) The mapping $t \longmapsto f(t, u, v)$ is measurable for any $(u, v) \in(0, \infty) \times \mathbb{R}$, and the mapping $(u, v) \longmapsto f(t, u, v)$ is continuous for almost every $t \in[0,1]$;

(b1) $\left|f\left(t, u_{1}, v\right)\right| \geq\left|f\left(t, u_{2}, v\right)\right|$ for almost every $t \in[0,1]$, for any $u_{1}, u_{2} \in(0, \infty)$ with $u_{1} \leq u_{2}$ and for any $v \in \mathbb{R}$

(b4) $\left|f\left(t, u, v_{1}\right)\right| \leq\left|f\left(t, u, v_{2}\right)\right|$ for almost every $t \in[0,1]$, for any $u \in(0, \infty)$ and for any $v_{1}, v_{2} \in \mathbb{R}$ with $v_{1} \leq v_{2}$

(c2) There exist $\alpha_{1} \in \mathbb{R}$ with $0<\alpha_{1}<\lambda$ and $\alpha_{2} \in \mathbb{R}$ with $\alpha_{2}>\lambda$ such that

$$
\int_{0}^{1}\left|f\left(t, \alpha_{1} t, \alpha_{2}\right)\right| d t<\infty
$$


(d1) There exists $\beta_{1} \in \mathbb{R}$ with $\beta_{1}>0$ such that

$$
\left|\frac{\partial f}{\partial u}(t, u, v)\right| \leq \frac{\beta_{1}|f(t, u, v)|}{u}
$$

for almost every $t \in[0,1]$, for any $u \in(0, \infty)$ and for any $v \in \mathbb{R}$;

(d2) There exists $\beta_{2} \in \mathbb{R}$ with $\beta_{2}>0$ such that

$$
\left|\frac{\partial f}{\partial v}(t, u, v)\right| \leq \beta_{2}|f(t, u, v)|
$$

for almost every $t \in[0,1]$, for any $u \in(0, \infty)$ and for any $v \in \mathbb{R}$;

(e) There exists the limit

$$
\lim _{t \rightarrow 0+} \frac{1}{t^{2}} \int_{0}^{t} s f\left(s, u(s), u^{\prime}(s)\right) d s
$$

for any continuously differentiable mapping $u$ from $[0,1]$ into $[0, \infty)$;

(f1) For $\alpha_{1}$ and $\alpha_{2}$,

$$
\lim _{t \rightarrow 0+} \frac{1}{t^{2}} \int_{0}^{t} s\left|f\left(s, \alpha_{1} s, \alpha_{2}\right)\right| d s=0
$$

Then there exists $h \in \mathbb{R}$ with $0<h \leq 1$ such that Cauchy problem (3) has a unique solution in $X$, where $X$ is a subset

$$
X=\left\{\begin{array}{l|l}
u & \begin{array}{l}
u \in C^{1}[0, h], u(0)=0, u^{\prime}(0)=\lambda, \\
\alpha_{1} t \leq u(t) \text { and } u^{\prime}(t) \leq \alpha_{2} \text { for any } t \in[0, h] \\
\text { and there exists the limit } \lim _{t \rightarrow 0+} \frac{t u^{\prime}(t)-u(t)}{t^{2}}
\end{array}
\end{array}\right\}
$$

of $C^{1}[0, h]$.

Proof By condition (c2), there exists $h_{1} \in \mathbb{R}$ with $0<h_{1} \leq 1$ such that

$$
\int_{0}^{h_{1}}\left|f\left(t, \alpha_{1} t, \alpha_{2}\right)\right| d t<\min \left\{\lambda-\alpha_{1}, \alpha_{2}-\lambda,\left(\frac{\beta_{1}}{\alpha_{1}}+2 \beta_{2}\right)^{-1}\right\} .
$$

By condition (f1), there exists $h \in \mathbb{R}$ with $0<h \leq h_{1}$ such that

$$
\sup _{t \in(0, h]} \frac{1}{t^{2}} \int_{0}^{t} s\left|f\left(s, \alpha_{1} s, \alpha_{2}\right)\right| d s \leq \int_{0}^{h_{1}}\left|f\left(t, \alpha_{1} t, \alpha_{2}\right)\right| d t .
$$

Let $A$ be an operator from $X$ into $C^{1}[0, h]$ defined by

$$
A u(t)=\lambda t+\int_{0}^{t}(t-s) f\left(s, u(s), u^{\prime}(s)\right) d s .
$$

Since a mapping $t \longmapsto \lambda t$ belongs to $X, X \neq \emptyset$. Moreover, we have $A(X) \subset X$. Indeed, by condition (a), $A u \in C^{1}[0, h], A u(0)=0$ and

$$
(A u)^{\prime}(0)=\left[\lambda+\int_{0}^{t} f\left(s, u(s), u^{\prime}(s)\right) d s\right]_{t=0}=\lambda
$$


By conditions (b1) and (b4), we obtain that

$$
\begin{aligned}
A u(t) & =\lambda t+\int_{0}^{t}(t-s) f\left(s, u(s), u^{\prime}(s)\right) d s \\
& \geq \lambda t-t \int_{0}^{h}\left|f\left(s, u(s), u^{\prime}(s)\right)\right| d s \\
& \geq \lambda t-t \int_{0}^{h}\left|f\left(s, \alpha_{1} s, \alpha_{2}\right)\right| d s \\
& \geq \alpha_{1} t
\end{aligned}
$$

and

$$
\begin{aligned}
(A u)^{\prime}(t) & =\lambda+\int_{0}^{t} f\left(s, u(s), u^{\prime}(s)\right) d s \\
& \leq \lambda+\int_{0}^{h}\left|f\left(s, u(s), u^{\prime}(s)\right)\right| d s \\
& \leq \lambda+\int_{0}^{h}\left|f\left(s, \alpha_{1} s, \alpha_{2}\right)\right| d s \\
& \leq \alpha_{2}
\end{aligned}
$$

for any $t \in[0, h]$. Moreover, by condition (e), there exists the limit

$$
\lim _{t \rightarrow 0+} \frac{t(A u)^{\prime}(t)-A u(t)}{t^{2}}=\lim _{t \rightarrow 0+} \frac{1}{t^{2}} \int_{0}^{t} s f\left(s, u(s), u^{\prime}(s)\right) d s
$$

We will find a fixed point of $A$. Let $\varphi$ be an operator from $X$ into $C^{1}[0, h]$ defined by

$$
\varphi[u](t)= \begin{cases}\frac{u(t)}{t} & \text { if } t \in(0, h] \\ \lambda & \text { if } t=0\end{cases}
$$

and

$$
\begin{aligned}
\varphi[X] & =\{\varphi[u] \mid u \in X\} \\
& =\left\{\begin{array}{l|l}
v \mid \begin{array}{l}
v \in C^{1}[0, h], v(0)=\lambda, \\
\alpha_{1} \leq v(t) \text { and } v(t)+t v^{\prime}(t) \leq \alpha_{2} \text { for any } t \in[0, h]
\end{array}
\end{array}\right\} .
\end{aligned}
$$

Then $\varphi[X]$ is a closed subset of $C^{1}[0, h]$ and hence it is a complete metric space. Let $\Phi$ be an operator from $\varphi[X]$ into $\varphi[X]$ defined by

$$
\Phi \varphi[u]=\varphi[A u]
$$

Then we can show, just like Theorem 2.1, that by the Banach fixed point theorem there exists a unique mapping $\varphi[u] \in \varphi[X]$ such that $\Phi \varphi[u]=\varphi[u]$ and hence $A u=u$.

Next, we consider the case of (III). 
Theorem 2.3 Let $\lambda$ be a real number with $\lambda>0$. Suppose that a mapping $f$ from $[0,1] \times$ $(0, \infty) \times \mathbb{R}$ into $\mathbb{R}$ satisfies the following:

(a) The mapping $t \longmapsto f(t, u, v)$ is measurable for any $(u, v) \in(0, \infty) \times \mathbb{R}$, and the mapping $(u, v) \longmapsto f(t, u, v)$ is continuous for almost every $t \in[0,1]$;

(b2) $\left|f\left(t, u_{1}, v\right)\right| \leq\left|f\left(t, u_{2}, v\right)\right|$ for almost every $t \in[0,1]$, for any $u_{1}, u_{2} \in(0, \infty)$ with $u_{1} \leq u_{2}$ and for any $v \in \mathbb{R}$

(b3) $\left|f\left(t, u, v_{1}\right)\right| \geq\left|f\left(t, u, v_{2}\right)\right|$ for almost every $t \in[0,1]$, for any $u \in(0, \infty)$ and for any $v_{1}, v_{2} \in \mathbb{R}$ with $v_{1} \leq v_{2}$

(c3) There exist $\alpha_{1} \in \mathbb{R}$ with $0<\alpha_{1}<\lambda$ and $\alpha_{2} \in \mathbb{R}$ with $\alpha_{2}<\lambda$ such that

$$
\int_{0}^{1}\left|f\left(t,\left(2 \lambda-\alpha_{1}\right) t, \alpha_{2}\right)\right| d t<\infty
$$

(d1) There exists $\beta_{1} \in \mathbb{R}$ with $\beta_{1}>0$ such that

$$
\left|\frac{\partial f}{\partial u}(t, u, v)\right| \leq \frac{\beta_{1}|f(t, u, v)|}{u}
$$

for almost every $t \in[0,1]$, for any $u \in(0, \infty)$ and for any $v \in \mathbb{R}$;

(d2) There exists $\beta_{2} \in \mathbb{R}$ with $\beta_{2}>0$ such that

$$
\left|\frac{\partial f}{\partial v}(t, u, v)\right| \leq \beta_{2}|f(t, u, v)|
$$

for almost every $t \in[0,1]$, for any $u \in(0, \infty)$ and for any $v \in \mathbb{R}$;

(e) There exists the limit

$$
\lim _{t \rightarrow 0+} \frac{1}{t^{2}} \int_{0}^{t} s f\left(s, u(s), u^{\prime}(s)\right) d s
$$

for any continuously differentiable mapping $u$ from $[0,1]$ into $[0, \infty)$;

(f2) For $\alpha_{1}$ and $\alpha_{2}$,

$$
\lim _{t \rightarrow 0+} \frac{1}{t^{2}} \int_{0}^{t} s\left|f\left(s,\left(2 \lambda-\alpha_{1}\right) s, \alpha_{2}\right)\right| d s=0
$$

Then there exists $h \in \mathbb{R}$ with $0<h \leq 1$ such that Cauchy problem (3) has a unique solution in $X$, where $X$ is a subset

$$
X=\left\{\begin{array}{l|l}
u & \begin{array}{l}
u \in C^{1}[0, h], u(0)=0, u^{\prime}(0)=\lambda, \\
\alpha_{1} t \leq u(t) \leq\left(2 \lambda-\alpha_{1}\right) t \text { and } \alpha_{2} \leq u^{\prime}(t) \text { for any } t \in[0, h] \\
\text { and there exists the limit } \lim _{t \rightarrow 0+} \frac{t u^{\prime}(t)-u(t)}{t^{2}}
\end{array}
\end{array}\right\}
$$

of $C^{1}[0, h]$.

Proof By condition (c3), there exists $h_{1} \in \mathbb{R}$ with $0<h_{1} \leq 1$ such that

$$
\int_{0}^{h_{1}}\left|f\left(t,\left(2 \lambda-\alpha_{1}\right) t, \alpha_{2}\right)\right| d t<\min \left\{\lambda-\alpha_{1}, \lambda-\alpha_{2},\left(\frac{\beta_{1}}{\alpha_{1}}+2 \beta_{2}\right)^{-1}\right\} .
$$


By condition (f2), there exists $h \in \mathbb{R}$ with $0<h \leq h_{1}$ such that

$$
\sup _{t \in(0, h]} \frac{1}{t^{2}} \int_{0}^{t} s\left|f\left(s,\left(2 \lambda-\alpha_{1}\right) s, \alpha_{2}\right)\right| d s \leq \int_{0}^{h_{1}}\left|f\left(t,\left(2 \lambda-\alpha_{1}\right) t, \alpha_{2}\right)\right| d t .
$$

Let $A$ be an operator from $X$ into $C^{1}[0, h]$ defined by

$$
A u(t)=\lambda t+\int_{0}^{t}(t-s) f\left(s, u(s), u^{\prime}(s)\right) d s .
$$

Since a mapping $t \longmapsto \lambda t$ belongs to $X, X \neq \emptyset$. Moreover, $A(X) \subset X$. Indeed, by condition (a), $A u \in C^{1}[0, h], A u(0)=0$,

$$
(A u)^{\prime}(0)=\left[\lambda+\int_{0}^{t} f\left(s, u(s), u^{\prime}(s)\right) d s\right]_{t=0}=\lambda
$$

by conditions (b2) and (b3),

$$
\begin{aligned}
A u(t) & =\lambda t+\int_{0}^{t}(t-s) f\left(s, u(s), u^{\prime}(s)\right) d s \\
& \geq \lambda t-t \int_{0}^{h}\left|f\left(s, u(s), u^{\prime}(s)\right)\right| d s \\
& \geq \lambda t-t \int_{0}^{h}\left|f\left(s,\left(2 \lambda-\alpha_{1}\right) s, \alpha_{2}\right)\right| d s \\
& \geq \alpha_{1} t, \\
A u(t) & =\lambda t+\int_{0}^{t}(t-s) f\left(s, u(s), u^{\prime}(s)\right) d s \\
& \leq \lambda t+t \int_{0}^{h}\left|f\left(s, u(s), u^{\prime}(s)\right)\right| d s \\
& \leq \lambda t+t \int_{0}^{h}\left|f\left(s,\left(2 \lambda-\alpha_{1}\right) s, \alpha_{2}\right)\right| d s \\
& \leq\left(2 \lambda-\alpha_{1}\right) t, \\
(A u)^{\prime}(t) & =\lambda+\int_{0}^{t} f\left(s, u(s), u^{\prime}(s)\right) d s \\
& \geq \lambda-\int_{0}^{h}\left|f\left(s, u(s), u^{\prime}(s)\right)\right| d s \\
& \geq \lambda-\int_{0}^{h}\left|f\left(s,\left(2 \lambda-\alpha_{1}\right) s, \alpha_{2}\right)\right| d s \\
& \geq \alpha_{2}
\end{aligned}
$$

for any $t \in[0, h]$, and by condition (e), there exists the limit

$$
\lim _{t \rightarrow 0+} \frac{t(A u)^{\prime}(t)-A u(t)}{t^{2}}=\lim _{t \rightarrow 0+} \frac{1}{t^{2}} \int_{0}^{t} s f\left(s, u(s), u^{\prime}(s)\right) d s
$$


We will find a fixed point of $A$. Let $\varphi$ be an operator from $X$ into $C^{1}[0, h]$ defined by

$$
\varphi[u](t)= \begin{cases}\frac{u(t)}{t} & \text { if } t \in(0, h] \\ \lambda & \text { if } t=0\end{cases}
$$

and

$$
\begin{aligned}
& \varphi[X]=\{\varphi[u] \mid u \in X\} \\
& =\left\{\begin{array}{l|l}
v & \begin{array}{l}
v \in C^{1}[0, h], v(0)=\lambda, \\
\alpha_{1} \leq v(t) \leq 2 \lambda-\alpha_{1} \text { and } \alpha_{2} \leq v(t)+t v^{\prime}(t) \text { for any } t \in[0, h]
\end{array}
\end{array}\right\} .
\end{aligned}
$$

Then $\varphi[X]$ is a closed subset of $C^{1}[0, h]$, and hence it is a complete metric space. Let $\Phi$ be an operator from $\varphi[X]$ into $\varphi[X]$ defined by

$$
\Phi \varphi[u]=\varphi[A u] .
$$

Then we can show, just like Theorem 2.1, that by the Banach fixed point theorem there exists a unique mapping $\varphi[u] \in \varphi[X]$ such that $\Phi \varphi[u]=\varphi[u]$ and hence $A u=u$.

Finally, we consider the case of (IV).

Theorem 2.4 Let $\lambda$ be a real number with $\lambda>0$. Suppose that a mapping $f$ from $[0,1] \times$ $(0, \infty) \times \mathbb{R}$ into $\mathbb{R}$ satisfies the following:

(a) The mapping $t \longmapsto f(t, u, v)$ is measurable for any $(u, v) \in(0, \infty) \times \mathbb{R}$, and the mapping $(u, v) \longmapsto f(t, u, v)$ is continuous for almost every $t \in[0,1]$;

(b2) $\left|f\left(t, u_{1}, v\right)\right| \leq\left|f\left(t, u_{2}, v\right)\right|$ for almost every $t \in[0,1]$, for any $u_{1}, u_{2} \in(0, \infty)$ with $u_{1} \leq u_{2}$ and for any $v \in \mathbb{R}$

(b4) $\left|f\left(t, u, v_{1}\right)\right| \leq\left|f\left(t, u, v_{2}\right)\right|$ for almost every $t \in[0,1]$, for any $u \in(0, \infty)$ and for any $v_{1}, v_{2} \in \mathbb{R}$ with $v_{1} \leq v_{2}$

(c4) There exist $\alpha_{1} \in \mathbb{R}$ with $0<\alpha_{1}<\lambda$ and $\alpha_{2} \in \mathbb{R}$ with $\alpha_{2}>\lambda$ such that

$$
\int_{0}^{1}\left|f\left(t,\left(2 \lambda-\alpha_{1}\right) t, \alpha_{2}\right)\right| d t<\infty
$$

(d1) There exists $\beta_{1} \in \mathbb{R}$ with $\beta_{1}>0$ such that

$$
\left|\frac{\partial f}{\partial u}(t, u, v)\right| \leq \frac{\beta_{1}|f(t, u, v)|}{u}
$$

for almost every $t \in[0,1]$, for any $u \in(0, \infty)$ and for any $v \in \mathbb{R}$;

(d2) There exists $\beta_{2} \in \mathbb{R}$ with $\beta_{2}>0$ such that

$$
\left|\frac{\partial f}{\partial v}(t, u, v)\right| \leq \beta_{2}|f(t, u, v)|
$$

for almost every $t \in[0,1]$, for any $u \in(0, \infty)$ and for any $v \in \mathbb{R}$; 
(e) There exists the limit

$$
\lim _{t \rightarrow 0+} \frac{1}{t^{2}} \int_{0}^{t} s f\left(s, u(s), u^{\prime}(s)\right) d s
$$

for any continuously differentiable mapping $u$ from $[0,1]$ into $[0, \infty)$;

(f2) For $\alpha_{1}$ and $\alpha_{2}$,

$$
\lim _{t \rightarrow 0+} \frac{1}{t^{2}} \int_{0}^{t} s\left|f\left(s,\left(2 \lambda-\alpha_{1}\right) s, \alpha_{2}\right)\right| d s=0 .
$$

Then there exists $h \in \mathbb{R}$ with $0<h \leq 1$ such that Cauchy problem (3) has a unique solution in $X$, where $X$ is a subset

$$
X=\left\{\begin{array}{ll}
u & \begin{array}{l}
u \in C^{1}[0, h], u(0)=0, u^{\prime}(0)=\lambda, \\
\alpha_{1} t \leq u(t) \leq\left(2 \lambda-\alpha_{1}\right) t \text { and } u^{\prime}(t) \leq \alpha_{2} \text { for any } t \in[0, h] \\
\text { and there exists the limit } \lim _{t \rightarrow 0+} \frac{t u^{\prime}(t)-u(t)}{t^{2}}
\end{array}
\end{array}\right\}
$$

of $C^{1}[0, h]$.

Proof By condition (c4), there exists $h_{1} \in \mathbb{R}$ with $0<h_{1} \leq 1$ such that

$$
\int_{0}^{h_{1}}\left|f\left(t,\left(2 \lambda-\alpha_{1}\right) t, \alpha_{2}\right)\right| d t<\min \left\{\lambda-\alpha_{1}, \alpha_{2}-\lambda,\left(\frac{\beta_{1}}{\alpha_{1}}+2 \beta_{2}\right)^{-1}\right\} .
$$

By condition (f2), there exists $h \in \mathbb{R}$ with $0<h \leq h_{1}$ such that

$$
\sup _{t \in(0, h]} \frac{1}{t^{2}} \int_{0}^{t} s\left|f\left(s,\left(2 \lambda-\alpha_{1}\right) s, \alpha_{2}\right)\right| d s \leq \int_{0}^{h_{1}}\left|f\left(t,\left(2 \lambda-\alpha_{1}\right) t, \alpha_{2}\right)\right| d t .
$$

Let $A$ be an operator from $X$ into $C^{1}[0, h]$ defined by

$$
A u(t)=\lambda t+\int_{0}^{t}(t-s) f\left(s, u(s), u^{\prime}(s)\right) d s .
$$

Since a mapping $t \longmapsto \lambda t$ belongs to $X, X \neq \emptyset$. Moreover, $A(X) \subset X$. Indeed, by condition (a), $A u \in C^{1}[0, h], A u(0)=0$,

$$
(A u)^{\prime}(0)=\left[\lambda+\int_{0}^{t} f\left(s, u(s), u^{\prime}(s)\right) d s\right]_{t=0}=\lambda,
$$

by conditions (b2) and (b4),

$$
\begin{aligned}
A u(t) & =\lambda t+\int_{0}^{t}(t-s) f\left(s, u(s), u^{\prime}(s)\right) d s \\
& \geq \lambda t-t \int_{0}^{h}\left|f\left(s, u(s), u^{\prime}(s)\right)\right| d s \\
& \geq \lambda t-t \int_{0}^{h}\left|f\left(s,\left(2 \lambda-\alpha_{1}\right) s, \alpha_{2}\right)\right| d s \\
& \geq \alpha_{1} t,
\end{aligned}
$$




$$
\begin{aligned}
A u(t) & =\lambda t+\int_{0}^{t}(t-s) f\left(s, u(s), u^{\prime}(s)\right) d s \\
& \leq \lambda t+t \int_{0}^{h}\left|f\left(s, u(s), u^{\prime}(s)\right)\right| d s \\
& \leq \lambda t+t \int_{0}^{h}\left|f\left(s,\left(2 \lambda-\alpha_{1}\right) s, \alpha_{2}\right)\right| d s \\
& \leq\left(2 \lambda-\alpha_{1}\right) t, \\
(A u)^{\prime}(t) & =\lambda+\int_{0}^{t} f\left(s, u(s), u^{\prime}(s)\right) d s \\
& \leq \lambda+\int_{0}^{h}\left|f\left(s, u(s), u^{\prime}(s)\right)\right| d s \\
& \leq \lambda+\int_{0}^{h}\left|f\left(s, \alpha_{1} s, \alpha_{2}\right)\right| d s \\
& \leq \alpha_{2}
\end{aligned}
$$

for any $t \in[0, h]$, and by condition (e), there exists the limit

$$
\lim _{t \rightarrow 0+} \frac{t(A u)^{\prime}(t)-A u(t)}{t^{2}}=\lim _{t \rightarrow 0+} \frac{1}{t^{2}} \int_{0}^{t} s f\left(s, u(s), u^{\prime}(s)\right) d s
$$

We will find a fixed point of $A$. Let $\varphi$ be an operator from $X$ into $C^{1}[0, h]$ defined by

$$
\varphi[u](t)= \begin{cases}\frac{u(t)}{t} & \text { if } t \in(0, h] \\ \lambda & \text { if } t=0\end{cases}
$$

and

$$
\begin{aligned}
\varphi[X] & =\{\varphi[u] \mid u \in X\} \\
& =\left\{\begin{array}{l|l}
v \mid \begin{array}{l}
v \in C^{1}[0, h], v(0)=\lambda, \\
\alpha_{1} \leq v(t) \leq 2 \lambda-\alpha_{1} \text { and } v(t)+t v^{\prime}(t) \leq \alpha_{2} \text { for any } t \in[0, h]
\end{array}
\end{array}\right\} .
\end{aligned}
$$

Then $\varphi[X]$ is a closed subset of $C^{1}[0, h]$ and hence it is a complete metric space. Let $\Phi$ be an operator from $\varphi[X]$ into $\varphi[X]$ defined by

$$
\Phi \varphi[u]=\varphi[A u]
$$

Then we can show, just like Theorem 2.1, that by the Banach fixed point theorem there exists a unique mapping $\varphi[u] \in \varphi[X]$ such that $\Phi \varphi[u]=\varphi[u]$ and hence $A u=u$. 


\section{Author details}

${ }^{1}$ College of Engineering, Nihon University, Fukushima, 963-8642, Japan. ${ }^{2}$ Marine Faculty of Engineering, Tamagawa University, Tokyo, 194-8610, Japan

Received: 1 June 2013 Accepted: 20 September 2013 Published: 07 Nov 2013

\section{References}

1. Knežević-Miljanović, J: On the Cauchy problem for an Emden-Fowler equation. Differ. Equ. 45(2), $267-270$ (2009)

2. Kawasaki, T, Toyoda, M: Existence of positive solution for the Cauchy problem for an ordinary differential equation. In: Li, S, Wang, X, Okazaki, Y, Kawabe, J, Murofushi, T, Guan, L (eds.) Nonlinear Mathematics for Uncertainly and Its Applications. Advances in Intelligent and Soft Computing, vol. 100, pp. 435-441. Springer, Berlin (2011)

10.1186/1029-242X-2013-465

Cite this article as: Kawasaki and Toyoda: Existence of positive solutions of the Cauchy problem for a second-order differential equation. Journal of Inequalities and Applications 2013, 2013:465

\section{Submit your manuscript to a SpringerOpen ${ }^{\circ}$ journal and benefit from:}

- Convenient online submission

- Rigorous peer review

- Immediate publication on acceptance

Open access: articles freely available online

- High visibility within the field

- Retaining the copyright to your article 